\title{
Sucrose intake functions of rat and cockroach for single and six solution presentations'
}

DONALD H. OWINGS, HERWALT A, HAERER AND ROBERT B. LOCKARD

UNIVERSITY OF WASHINGTON

Intake of sucrose solution was studied in a comparative experiment in which roaches and rats were given access to one or to six concentrations for a single $23 \mathrm{hr}$. test. Intake was an inverted-U shaped function of concentration in the single bottle test and a monotonic increasing function in the six bottle test. The relevance of choice behavior was discussed in relation to the multiple bottle procedure.

Functions relating intake of sucrose solutions to concentration typically show maximum intake at about 8\% (Richter \& Campbell, 1940; Young, 1966). Such inverted- $U$ shaped functions are obtained in procedures in which rats are allowed free access to only a single concentration of sucrose at a time. On the other hand, other evidence suggests that the function relating intake to concentration becomes monotonic when rats are allowed free access to two or more concentrations simultaneously. This evidence is, however, confined to two concentrations (Young \& Greene, 1953), and to a study by Carpenter (1958), whose use of groups of rats with access to a common fluid source raises methodological problems. In view of the limited nature of this evidence, an attempt was made to obtain both the monotonic and inverted- $U$ function in the same experiment by giving individual rats access to one or to six sucrose solutions.

The disparity between the functions obtained in single versus multiple solution presentations has been attributed to the absence or presence of an opportunity to display choice behavior (Young, 1966). A second objective, then, was to determine whether the disparity between the functions could be reduced by using an animal considered less capable than the rat of learning and, therefore, of displaying choice behavior. The cockroach (Periplaneta americana) was chosen because it is far removed phyletically from the rat, has been shown to accept sucrose (Frings, 1946), and has greater daily fluid intake than the more thoroughly studied blowfly (Dethier, 1964).

\section{Experiment l.}

The rats used in the one-bottle procedure were 29 120-day-old Holtzman albinos. Those used in the sixbottle procedure were eight 90-day-old Holtzman albinos.

The Ss were maintained in individual stainless steel cages (16 in. $x 10$ in. $x 7$ in.) with wire mesh floors and fronts. Ss in the one-bottle procedure were tested in their maintenance cages. Those in the six-bottle procedure were moved to different but identical cages for testing, since they were tested four at a time. Fluid was presented in glass drinking bottles with stainless steel tubes extending $1 / 4$ in. into the cage and $1-1 / 2$ in. above the floor. The drinking apertures were centered on the front of the cage for the onebottle procedure and were spaced $1-1 / 2$ in. apart for the six-bottle procedure. Cage illumination was about $1.2 \mathrm{ft} .-\mathrm{c}$. Solutions were weighed on a balance accurate to $0.1 \mathrm{gm}$. Ss were maintained on a $12 \mathrm{~L}: 12 \mathrm{D}$ light-dark cycle.

Concentrations of sucrose used were 0 (distilled water), $2,4,8,16$, and $32 \%$ by weight of solute and solvent; e.g., $4 \%$ is $4 \mathrm{gm}$ sugar in $96 \mathrm{gm}$ water (Pfaffman et al, 1954). All solutions were mixed with distilled water and were used only at room temperature. Fresh solutions were used for each test, and were allowed to stand at least $3 \mathrm{hr}$. before use.

For the one-bottle test, each rat was exposed to only a single concentration of sucrose solution. Five rats were assigned at random to each concentration (0-16) and four to the $32 \%$ solution. Intake of solution was measured for a single $23 \mathrm{hr}$. period. No water was available during testing, but Purina rat pellets were available ad lib. In the six-bottle test, each rat was exposed simultaneously to $0,2,4,8,16$, and $32 \%$ concentrations of sucrose solution for a single $23 \mathrm{hr}$. period. The spatial order of the solutions was random. Experiment II.

Ss were 15 adult male and female cockroaches (Periplaneta americana) from a commercial Florida source. Ss were maintained and tested in individual black plastic boxes ( 3 in. $x 4$ in. $\times 1$ in.) with transparent tops, guillotine doors and six 3/16-in. holes 1/4 in. apart. A 12L:12D light-dark cycle was maintained throughout the maintenance and test periods. Purina rat chow and distilled water were available ad lib with the exception that water was not available on single solution test days.

Solutions of $0,2,4,8,16$, and $32 \%$ were prepared as above. The solutions were delivered via $1 \mathrm{ml}$ serological pipettes (graduated to $0.01 \mathrm{ml}$ ) inclined $5 \mathrm{de}$ grees from the horizontal. In the six-solution test, Ss were tested individually in their home cages by introducing the pipettes into the six holes. Solutions were randomized with respect to position. In the single solution test, two Ss were assigned to each concentration and subsequently received an ascending or descending series with one day of distilled water between test days. All test sessions lasted $23 \mathrm{hr}$. Resulis

Intake functions for rats and roaches are presented in Fig. 1. Single-bottle intake of solutions was found to vary significantly as a function of concentration for 


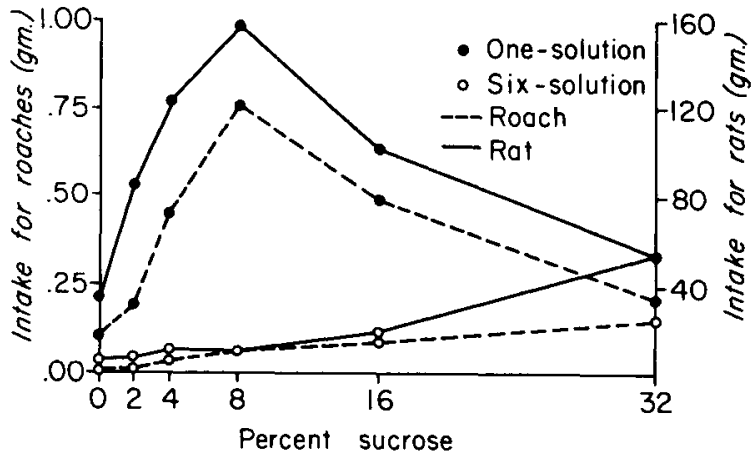

Fig. 1. Mean sucrose solution intake as a function of concentration for rats and roaches in one and six bottle procedures.

both rats and roaches $(F=19.9, \mathrm{df}=5 / 23, \mathrm{p}<.005$; $F=24.2, d f=5 / 30, p<.005$, respectively), with sequential effects for roaches quite insignificant. In the six-bottle procedure a Friedman two-way analysis of variance showed that differential intake of solutions was significant for both rats and roaches $\left(\mathrm{X}_{\mathrm{r}}^{2}=21.5\right.$, $\mathrm{df}=5, \mathrm{p}<.001 ; \mathrm{X}_{\mathrm{r}}^{2}=42.3, \mathrm{df}=5, \mathrm{p}<.001$, respectively). Discussion

The results clearly demonstrate that number of alternatives is a critical variable in the determination of the shape of sucrose intake functions for animals as phyletically distant as rats and roaches. That the variable is operative for roaches as well as rats makes questionable the hypothesis that choice behavior -use of cues not inherent in the solution-is necessary for the generation of the monotonic function. If choice behavior were necessary one would have hypothesized that the disparity between the two roach functions would have been much less than between the two rat functions since roaches have not been shown to possess the learning capacity exhibited by rats. It is possible, however, that rats display choice behavior in this situation and roaches do not. Observations of rats toward the end of the first hour of exposure suggested that some non-gustatory cue was being learned (i.e., selection of a particular solution appeared to occur without sampling). Roaches, on the other hand, appeared to sample throughout comparable time periods. It must be emphasized that these observations were mere samples during the first hour of testing; roaches may have demonstrated similar apparent learned behavior during subsequent hours of the test. It is possible, though, that roaches display sucrose intake behavior similar to that of the blowfly (Dethier, 1964); solutions may be contacted at random, intake deter- mined by concentration, and subsequent acceptance a function of the concentration previously ingested. For example, contact and intake of $8 \%$ would reduce the probability of subsequent acceptance of the same or lower concentrations for some fraction of the 23 $\mathrm{hr}$. test. Such behavior would suffice to generate the monotonic function in multiple bottle tests. It is important to note the possibility that the factor used by most psychologists to account for the descending arm of the inverted-U function-satiation, or post-ingestion -is a critical factor in the generation of the monotonic intake function. This points to the possibility that the monotonic function generated in intake tests may be only slightly related to the monotonic functions that relate some instrumental response measure to concentration. Although the latter may be attributable solely to the stimulating properties of sucrose (taste), the former may result from a change in probability of acceptance as a result of ingestion.

In conclusion, it is not clear whether the similarity in the rat and roach results reflects homologous, or merely analogous behavioral mechanisms. The data strongly confirm the importance of the number of alternatives variable in sucrose intake procedures and suggest a pervasive analogy in the relative intake of sucrose solutions across phyla. The only noninferential statement that can be made about the difference in the single versus multiple presentation procedures is, however, that the single bottle procedure allows intake of a particular solution to affect subsequent intake of only that solution, whereas the multiple bottle procedure allows intake of a particular solution to affect subsequent intake of other solutions.

\section{References}

Carpenter, J. A. A comparison of stimulus-presentation procedures in taste-preference experiments. $J$, comp. physiol. Psychol., $1958,51,561-564$.

Dethier, V. G. Microscopic brains. Science, 1964, 143, 1138-1145.

Frings, H. Gustatory thresholds for sucrose and electrolytes for the cockroach Periplaneta americana (Linn.). J. exp. Zool., $1946,102,23-50$.

Richter, C. P., \& Campbell, K. N. Taste thresholds and taste preferences of rats for five common sugars. J. Nutrition, 1940, $20,31-40$

Young, P. T. Hedonic organization and the regulation of behavior. Psychol. Rev., 1966, 73, 59-86.

Young, P. T., \& Greene, J. T. Quantity of food injested as a miasure of relative acceptability. J. comp. physiol. Psychol, $1953,46,288-294$.

\section{Note}

1. This research was supported by Grant GB-3141 from the National Science Foundation. 